\title{
Contrôle optique pour fonctionnalités microondes
}

\author{
René Gary, Mohamed El Khaldi, Jean-Daniel Arnould, Florence Podevin et Anne Vilcot \\ Institut de Microélectronique, Electromagnétisme et Photonique, UNR 5130 INPG-UJF-CNRS, 23, \\ rue des Martyrs, BP 257, 38016 Grenoble, cedex 1
}

\begin{abstract}
Résumé - Deux axes sont envisagés ici. Le premier axe s'intéresse aux efforts de modélisation du plasma d'électrons et de trous photoinduits au sein d'un semiconducteur. Cette modélisation est effectuée dans un but de conception de prototypes. Les optimisations les plus récentes permettent de tenir compte de la forme du champ lumineux injecté et non plus de la seule puissance lumineuse mais surtout de poursuivre le calcul de façon semianalytique. Le deuxième axe présente une fonctionnalité microondes: le déphasage de fréquence. Le déphaseur a montré des performances intéressantes centrées autour de $10 \mathrm{GHz}, 90^{\circ}$ de déphasage sur une bande de $5 \%$, une adaptation de $-20 \mathrm{~dB}$ sur une bande de $20 \%$ et une variation du coefficient de transmission inférieur à $5 \mathrm{~dB}$ à 10 GHz.
\end{abstract}

Mots-clefs - contrôle optique, photoconductivité, déphasage, filtrage, reconfigurabilité.

\section{INTRODUCTION}

Le contrôle optique de composants microondess est une activité amont de l'Institut de Microélectronique, Electromagnétisme et Photonique. En éclairant du silicium hautement résistif ( $\mathrm{Si} \mathrm{HR}$ ), peu dopé, voire non dopé, à l'aide d'un signal lumineux de longueur d'onde appropriée, des porteurs sont photoinduits. Du fait de leur durée de vie de l'ordre de la microseconde dans le $\mathrm{Si}$ $\mathrm{HR}$, ils diffusent sur des longueurs de l'ordre de $100 \mu \mathrm{m}$. On parle de plasma photoinduit, que l'on peut modéliser dans une première approximation par une charge microondes complexe. Cette charge varie en fonction du champ lumineux appliqué. Si l'éclairement est judicieusement choisi au sein d'un composant microondess en technologie microruban ou coplanaire, la propagation d'une onde hyperfréquence dans ce composant est modifiée. On obtient ainsi des composants accordables optiquement, atténuateurs variables [1], filtres accordables [2], adaptateurs d'impédance [3], déphaseurs [4]. Le recours au contrôle optique trouve son intérêt dans la mesure où il n'est pas limité en fréquence, contrairement aux charges variables actuelles telles que les diodes ou les capacités varactors. Néanmoins, afin de démontrer la faisabilité des dispositifs et/ou de les comparer avec ceux existants, les fréquences de fonctionnement étudiées sont inférieures à $30 \mathrm{GHz}$.

Deux axes sont envisagés ici. Le premier axe s'intéresse aux efforts de modélisation effectués dans un but de conception des prototypes. Les optimisations les plus récentes permettent de calculer semi-analytiquement les densités de charges photoinduites prenant en compte toutes les caractéristiques physiques et géométriques de l'éclairement et du substrat. Le deuxième axe présente un exemple de fonctionnalité microondess : le déphasage de fréquence. Il s'agit d'un déphasage variable commandé optiquement pour des applications d'antennes à balayage de phase dans le but de contrôler la direction du faisceau hertzien. Des études en transmission sur des lignes à gap, des composants résonants et ceux dits de couplage ont permis de montrer que les structures couplées offraient le meilleur compromis et une grande latitude quant aux gammes de déphasage atteignables. Les études théoriques et expérimentales présentées concernent un déphaseur hyperfréquences basé sur le coupleur en anneau de Wilkinson. Il permet d'atteindre un déphasage continu de $90^{\circ}$ sur une bande de $500 \mathrm{MHz}$ autour de $10 \mathrm{GHz}$ sous une puissance optique de $30 \mathrm{~mW}$, avec une variation du coefficient de transmission de seulement $5 \mathrm{~dB}$ et une adaptation de $-20 \mathrm{~dB}$ sur une plage de fréquence de $20 \%$ autour de $9,8 \mathrm{GHz}$.

\section{MODELISATION DU PLASMA PHOTOINDUIT}

Le contrôle optique exploite l'effet photoconducteur qui consiste à convertir des photons de longueur d'onde appropriée en paires électrons-trous dans un substrat semiconducteur par une injection optique d'énergie supérieure à la bande interdite du substrat. La création d'un plasma photoinduit modifie simultanément la conductivité du substrat, ainsi que sa permittivité complexe, dans la région éclairée. Ce phénomène a été démontré pour la première fois sur un guide semiconducteur de très grande résistivité par $\mathrm{C}$. $\mathrm{H}$. Lee et al [5]. On trouvera une description détaillée des phénomènes mis en jeu dans [6].

Les équations suivantes, (1) à (3), permettent d'exprimer les constantes de propagation $\beta_{\mathrm{p}}$ et d'atténuation $\alpha_{\mathrm{p}}$, à partir de la donnée de la permittivité complexe $\varepsilon_{\mathrm{p}}$ présentée par le plasma. L'équation (1) donne la valeur de $\varepsilon_{\mathrm{p}}$ sous différentes conditions. On suppose que le plasma est uniforme, que le nombre de trous générés est identique au nombre d'électrons et que le nombre de porteurs excédentaires est supérieur au nombre de porteurs présents dans le semiconducteur sans éclairement. Les équations (2) et (3) indiquent la dépendance des constantes de propagation et 
d'atténuation en fonction de l'indice de réfraction complexe $\overline{\mathrm{n}}_{\mathrm{p}}$.

$$
\begin{gathered}
\varepsilon_{\mathrm{p}}=\varepsilon_{\mathrm{r}}-\left(\mathrm{m}_{\mathrm{e}} \mu_{\mathrm{e}}^{2}+\mathrm{m}_{\mathrm{h}} \mu_{\mathrm{h}}^{2}\right) \frac{\Delta \mathrm{n}}{\varepsilon_{0}}-\mathrm{j} \frac{\mathrm{e}\left(\mu_{\mathrm{e}}+\mu_{\mathrm{h}}\right) \Delta \mathrm{n}}{\varepsilon_{0} \omega} \\
\bar{n}_{\mathrm{p}}^{2}=\left(\mathrm{n}_{\mathrm{p}}-\mathrm{j} \kappa_{\mathrm{p}}\right)^{2}=\varepsilon_{\mathrm{p}} \\
\alpha_{\mathrm{p}}\left(\mathrm{m}^{-1}\right)=\frac{2 \pi \mathrm{f}}{\mathrm{c}} \kappa_{\mathrm{p}} \text { et } \beta_{\mathrm{p}}\left(\mathrm{m}^{-1}\right)=\frac{2 \pi \mathrm{f}}{\mathrm{c}} \mathrm{n}_{\mathrm{p}}
\end{gathered}
$$

La présence du plasma se traduit vis-à-vis d'un signal microondes se propageant dans le substrat par un changement de phase parallèlement à une atténuation contrôlables optiquement. Les deux phénomènes peuvent être mis à profit dans diverses fonctionnalités microondess, l'idéal étant de pouvoir s'affranchir de l'un ou de l'autre afin de bénéficier pleinement de la reconfigurabilité voulue.

Deux types de simulation ont été développés à l'IMEP. L'une fait appel à une résolution numérique, l'autre à un développement semi-analytique.

\section{A. Résolution numérique}

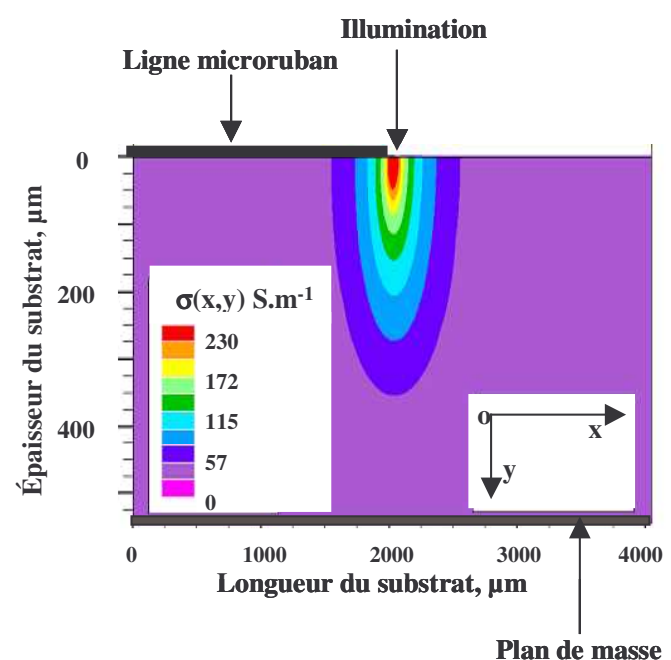

(a)



(b)

Fig. 1 Evolution de la conductivité du silicium éclairé sous une illumination de $250 \mu \mathrm{W}$, à l'extrémité d'une ligne microruban Schottky, sur Si HR $4000 \Omega . c m$, (a) diffusion latérale et en profondeur, (b), profil suivant la profondeur.
Le logiciel utilisé, Atlas de Silvaco 2D [7], fait appel à la résolution des équations de continuité du courant au sein du semiconducteur et permet de déterminer les concentrations de porteurs sous différentes conditions, de polarisation et/ou d'éclairement (avec le module Luminous), etc. La figure 1 représente le profil de conductivité obtenu sous un éclairement de $250 \mu \mathrm{W}$ à l'extrémité d'une ligne microruban à contact Schottky, au sein d'un substrat de silicium de résistivité $4000 \Omega$.cm, avec une vitesse de recombinaison surfacique nulle. Le type de contact métallique intervient sur le profil de conductivité. Dans le cas du contact ohmique, bien que les porteurs de type $\mathrm{p}$ s'accumulent à l'interface, la fuite de porteurs de type $n$ vers le métal induit un profil de conductivité beaucoup plus faible sous le métal qu'en volume, ce qui est un cas a priori défavorable dans un objectif de minimisation de la consommation en puissance lumineuse.

\section{B. Résolution analytique}

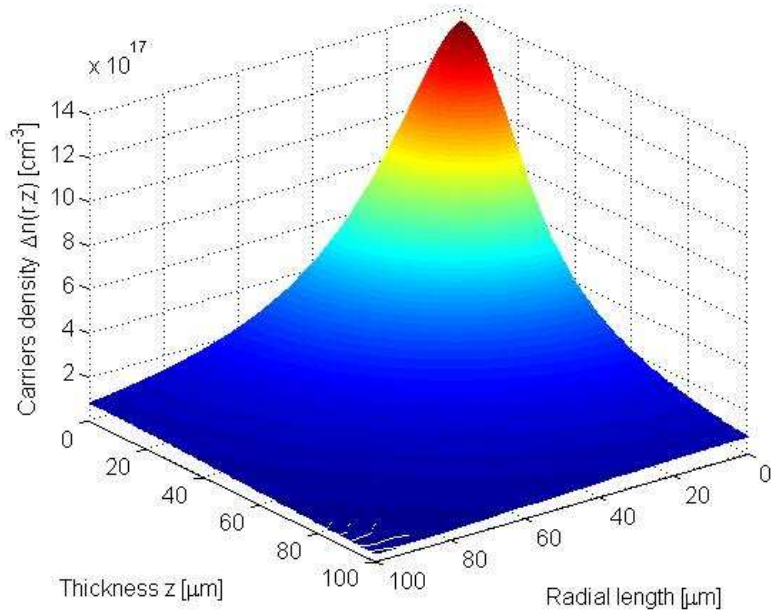

\begin{tabular}{|c|c|}
\hline Paramètres & Valeur \\
\hline Longueur d'onde $\lambda_{\text {lum }}$ & $830 \mathrm{~nm}$ \\
\hline $1 / 2$ Largeur faisceau $\mathrm{w}_{0}$ & $25 \mu \mathrm{m}$ \\
\hline Puissance lumineuse $P_{\text {lum }}$ & $0,1 \mathrm{~W}$ \\
\hline Coefficient d'absorption $\alpha$ & $0,085 \mu \mathrm{m}^{-1}$ \\
\hline Durée de vie $\tau_{0}=\tau_{\mathrm{n}}=\tau_{\mathrm{p}}$ & $2 \mu \mathrm{s}$ \\
\hline Coefficient de diffusion des $\mathrm{e}^{-} \mathrm{D}_{\mathrm{n}}$ & $34,9 \mathrm{~cm}^{2} \cdot \mathrm{s}^{-1}$ \\
\hline Coefficient de diffusion des trous $\mathrm{D}_{\mathrm{p}}$ & $12,4 \mathrm{~cm}^{2} \cdot \mathrm{s}^{-1}$ \\
\hline Concentration de trou intrinsèque $\mathrm{p}_{0}$ & $2,6.10^{12} \mathrm{~cm}^{-3}$ \\
\hline Concentration d'e- intrinsèque $\mathrm{n}_{0}$ & $3,8.10^{7} \mathrm{~cm}^{-3}$ \\
\hline $\begin{array}{l}\text { Vitesse de recombinaison surfacique } \\
\mathrm{s}_{0}\end{array}$ & $0 \mathrm{~cm} \cdot \mathrm{s}^{-1}$ \\
\hline
\end{tabular}

Fig 2. Représentation en 3D de la densité de porteurs photocréés.

\section{TABLEAU 1}

Récemment, ce calcul numérique de la densité de porteurs photocréés dans le substrat a fait l'objet d'une optimisation par un calcul semi-analytique. Les avantages de ce dernier sont la vitesse de calcul mais aussi et 
surtout la précision du calcul, ainsi qu'une meilleure appréhension des phénomènes physique mis en jeu.

La description précise et mathématique du calcul est largement détaillée dans [8], ainsi seule une rapide description sera développée ici. En partant des équations de continuité du courant, en y appliquant les principes d'électroneutralité et d'éclairement permanent, la somme des courants dans le substrat nous permet d'exprimer la densité de porteurs en 3D sous la forme de l'équation de diffusion ambipolaire. Cette équation du deuxième ordre à coefficients variables avec second membre est résolue par l'application de la transformée de Hankel. Cette transformée, qui n'est autre qu'une transformée de Fourier généralisée mais qui présente des propriétés intéressantes sur les symétries cylindriques, permet de passer de l'équation de diffusion à une équation différentielle du second ordre à coefficients constants qui peut être résolue de façon classique. L'application de la transformée inverse sur cette solution permet d'exprimer la densité de porteurs sous la forme d'une intégrale qui peut être calculée numériquement à l'aide de Matlab [9].

La figure 2 présente cette densité de porteurs en 3 dimensions dans un substrat silicium dont les caractéristiques sont résumées dans le tableau 1

\section{Simulations électromagnétiques}

Les profils obtenus numériquement ou analytiquement sont discrétisés sous forme d'ellipsoïdes et intégrés au sein du substrat dans le but d'une résolution EM numérique $3 \mathrm{D}$, comme présenté sur la figure 3. L'effet de la lumière est donc modélisé par l'introduction de ces couches actives définies par une permittivité complexe dépendant de la conductivité, c'est-à-dire du nombre de porteurs photoinduits, tel que dans l'équation (1). Les résultats présentés ici ont été obtenus à l'aide de CST Microwave Studio [10] qui utilise la technique des équations intégrales pour résoudre les équations de Maxwell. Cette technique est bien adaptée pour simuler des circuits planaires intégrés au sein d'une structure multicouche.

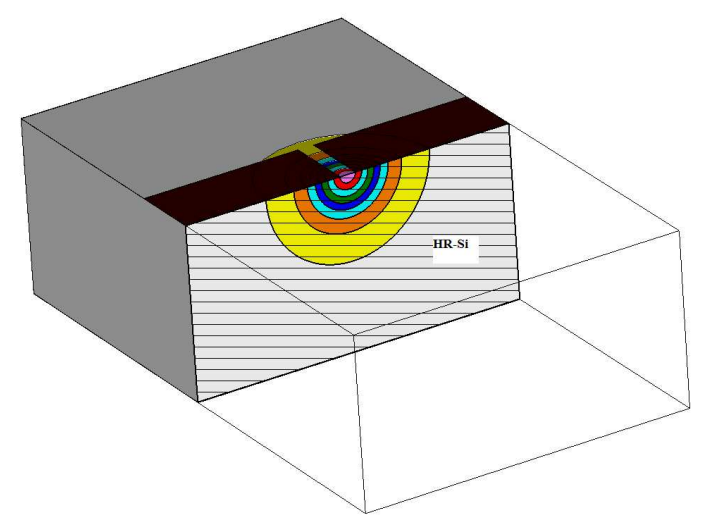

Fig. 3. Modèle 3D en coupe d'un gap photexcité, avec l'implémentation de 8 demi-sphères pour modéliser le plasma.

\section{APPLICATION : DEPHASEUR DE FREQUENCE}

Le modèle présenté au paragraphe précédent a été mis à profit afin de simuler le comportement de dispositifs microondess tels que les déphaseurs hyperfréquences ou les filtres à structure périodique accordables.

\section{A. Topologie et conception}

La structure proposée en technologie microruban $50 \Omega$ est présentée sur la figure 4. Il s'agit du coupleur en anneau de Wilkinson. Les lignes $50 \Omega$ ont une largeur de $443 \mu \mathrm{m}$ et sont déposées sur une tranche de silicium de résistivité $4000 \Omega . \mathrm{cm}$, de $540 \mu \mathrm{m}$ d'épaisseur. Entre les ports adaptés $1,2,3$ et 4 , en générant un signal microondess sur le port 1 , on ne reçoit rien sur le port 2 , en revanche sur les ports 3 et 4 , les ondes présentent une opposition de phase. Notre but est de transmettre la somme de ces deux ondes sur le port 2, en n'ayant aucune réflexion sur le port 1 . Dans ce but, le port 4 est allongé par une ligne quart d'onde $\left(\theta_{0}=\pi / 2\right.$ à $\left.10 \mathrm{GHz}\right)$ de telle sorte que les signaux réfléchis au niveau des gaps en 3 et 4' s'annulent en 1 et s'additionnent en 2.

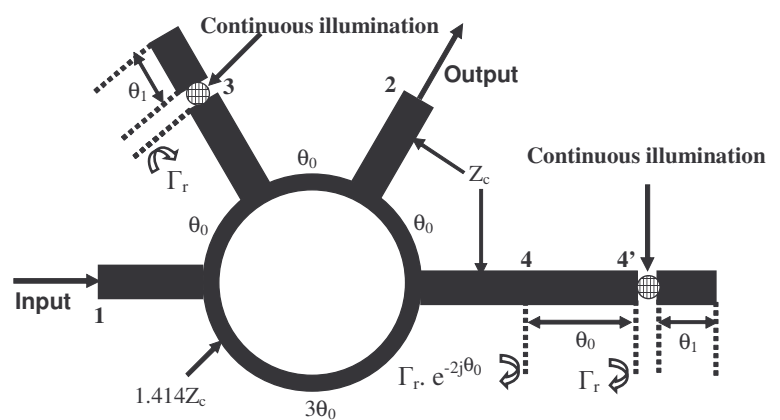

(a)

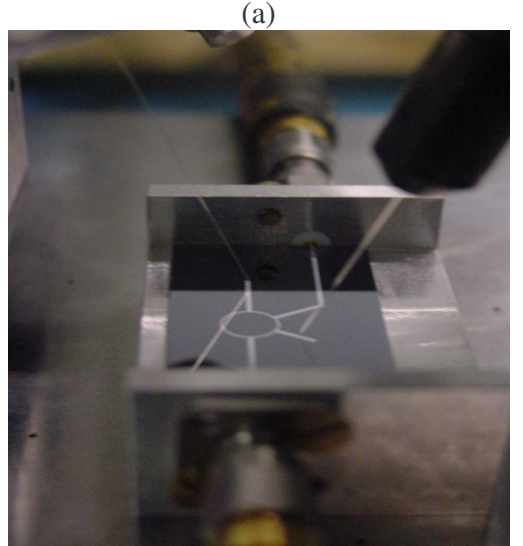

(b)

Fig. 4. Coupleur hybride de Wilkinson à gap pour une application de déphasage contrôlé optiquement à $10 \mathrm{GHz}$. $Z_{\mathrm{c}}=50 \Omega ; \theta_{0}=\pi / 2$ et $\theta_{1}=\pi / 4$ à $10 \mathrm{GHz}$, (a) vue de dessus, (b) composant expérimental.

La matrice $\left[S_{\mathrm{ij}}\right]$ du quadripôle entre les ports 1 et 2 peut être calculée à partir de celle de l'octopôle situé entre les ports $1,2,3$ et 4'. L'expression de ses paramètres $s_{i j}$ est facilement établie en utilisant la technique des modes pair et impairs [11] : 


$$
\begin{gathered}
s^{\prime}{ }_{11}=s^{\prime}{ }_{22}=s^{\prime}{ }_{33}=s^{\prime}{ }_{44}=0 ; \\
s^{\prime}{ }_{12}=s^{\prime}{ }_{21}=s^{\prime}{ }_{34}=s^{\prime}{ }_{43}=0 ; \\
s_{13}^{\prime}=s_{31}^{\prime}=s_{24}^{\prime}=s_{42}^{\prime}=s_{23}^{\prime}=s_{32}^{\prime}=\frac{-j}{\sqrt{2}} ; s_{14}^{\prime}=s_{41}^{\prime}=\frac{j}{\sqrt{2}}
\end{gathered}
$$

En tenant compte de la section supplémentaire de longueur électrique $\theta_{0}$, entre 4 et 4 ', et en négligeant les termes de réflexion d'ordre supérieur, il est possible de déterminer les paramètres de réflexion $S_{11}$ et de transmission $S_{21}$ ainsi :

$$
\begin{aligned}
& S_{11}=s_{11}^{\prime}+\Gamma_{r}\left(s_{13}^{\prime 2}+s_{14}^{\prime 2} e^{-2 j \theta_{0}}\right) \\
& S_{21}=s_{12}^{\prime}+\Gamma_{r} s_{13}^{\prime}\left(s_{23}^{\prime}+s_{14}^{\prime} e^{-2 j \theta_{0}}\right)
\end{aligned}
$$

Le coefficient de réflexion $\Gamma_{\mathrm{r}}$ est dû à l'illumination des gaps au niveau des ports 3 et 4'. A la fréquence centrale de $10 \mathrm{GHz}, \theta_{0}=\pi / 2, S_{11}=0$ et $S_{21}=-\Gamma_{\mathrm{r}}$. A l'obscurité, $\Gamma_{\mathrm{r}}=1$, soit $\mathrm{S}_{21}=\mathrm{e}^{\mathrm{j} \pi}$. Sous forte illumination (saturation), $\Gamma_{\mathrm{r}}=\mathrm{e}^{-\mathrm{j} 2 \theta 1}=\mathrm{e}^{-\mathrm{j} \pi / 2}$, soit $\mathrm{S}_{21}=\mathrm{e}^{\mathrm{j} \pi / 2}$. Ainsi le déphasage maximal théorique entre obscurité et forte illumination est contrôlé par la longueur électrique $\theta_{1}=45^{\circ}$ à $10 \mathrm{GHz}$. De plus la différence d'amplitude est nulle. Ceci est valable dans la mesure où le gap n'a que deux états (ON : métallique) ou (OFF : purement diélectrique).

En augmentant $\theta_{1}$, conjointement, on augmente le déphasage. $90^{\circ}$ nous ont semblé, toutefois, suffisants. Premièrement, en théorie, un déphasage de $90^{\circ}$ suffit à pouvoir conformer un faisceau dans un demi-espace, à condition que les antennes imprimées soient suffisamment espacées, d'environ un quart de la longueur d'onde en espace libre. Deuxièmement, les pertes par propagation diélectriques sont élevées sur silicium. Même un silicium d'une résistivité de $4000 \Omega . \mathrm{cm}$ présente des pertes diélectriques d'environ $0,35 \mathrm{~dB} / \mathrm{cm}$ avec notre topologie de lignes microruban. Réduire la longueur électrique limite donc les pertes et tend également vers une politique de plus grande intégration.

\section{B. Simulation et résultats expérimentaux}

La figure 5 donne les réponses en transmission et en réflexion simulées du composant de la figure 4.(a). L'illumination a été modélisée au moyen d'un spot cylindrique constant de diamètre $50 \mu \mathrm{m}$. Le déphasage de l'onde transmise dépend fortement du signal optique injecté (figure 5(a)). Le déphasage de $90^{\circ}$ à $10 \mathrm{GHz}$ a été obtenu sous une puissance optique de $30 \mathrm{~mW}$, avec une variation d'amplitude de seulement $5 \mathrm{~dB}$, qui peuvent aisément être compensés. Cette variation, alors qu'idéalement $\left|S_{21}\right|$ devrait rester pratiquement constant est due aux photoporteurs et à leur influence sur la partie imaginaire de la permittivité diélectrique complexe du plasma. La valeur de référence est de $2,5 \mathrm{~mW}$, au lieu de $0 \mathrm{~mW}$. En effet, avec un gap de $50 \mu \mathrm{m}$ de long, la sensibilité au contrôle optique était très faible en dessous de 2,5 $\mathrm{mW}$. Concernant les pertes de désadaptation, on remarque une adaptation meilleure que $20 \mathrm{~dB}$ entre 9 et $11 \mathrm{GHz}$. Autour de $10 \mathrm{GHz}$, sur $500 \mathrm{MHz}$ de bande, pour une illumination donnée, la variation de $\varphi\left(S_{21}\right)$ est inférieure à $5 \%$, c'est-à-dire suffisamment faible pour pouvoir être utilisée dans des communications dites large-bande.
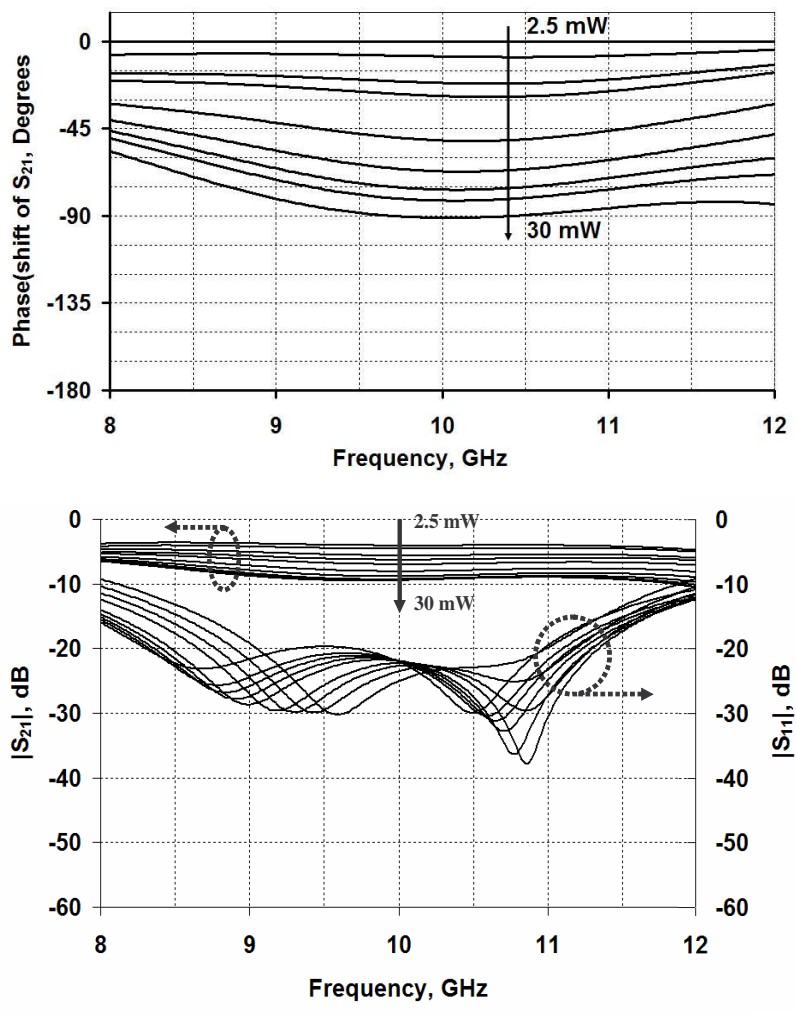

(a)

Fig. 5 Simulation (a) de la phase de $\mathrm{S}_{21}$ et (b) de l'amplitude de $S_{21}$ et de $S_{11}$ sous différentes conditions d'éclairement.

Pour les mesures, une diode laser SDL2320 $(\lambda=840 \mathrm{~nm})$ couplée à une fibre multimode a été utilisée. Elle fournit une puissance optique suffisante, $400 \mathrm{~mW}$ au maximum. La symétrie est assure au moyen d'un diviseur optique commercial. Le diamètre de cœur de la fibre est exactement de $50 \mu \mathrm{m}$ de sorte que la fibre est placée juste au dessus du gap. Les résultats de mesure sont représentés sur la figure 6 . Le déphasage de $90^{\circ}$ a été obtenu, tel qu'attendu. Quant au coefficient de transmission $\left|S_{21}\right|$, une variation d'environ $5 \mathrm{~dB}$ a été atteinte à $10 \mathrm{GHz}$, telle que prédite par la simulation. L'aspect large-bande de l'adaptation n'a pas été dégradé puisqu' on atteint $-20 \mathrm{~dB}$ entre $8,9 \mathrm{GHz}$ et $10,7 \mathrm{GHz}$. La constance de la phase avec la fréquence est toujours observée dans une bande de $5 \%$ autour de $10 \mathrm{GHz}$.

Remarquons que la puissance optique nécessaire pour atteindre le déphasage maximum est importante : $30 \mathrm{~mW}$. En effet, le gap de $50 \mu \mathrm{m}$ est large. Un gap plus court de $5 \mu \mathrm{m}$, le diamètre de cœur d'une fibre monomode, serait plus adapté et permettrait une réduction de la consommation. Parallèlement, le coefficient d'atténuation 
dû au gap illuminé, $\alpha$, aurait moins d'influence, ce qui induirait une réduction de $\Delta\left|S_{21}\right|$. Enfin, avec un gap plus court, la constante de phase, $\beta$, du gap illuminé aurait également moins d'influence. Ainsi le déphasage maximal serait contrôlé uniquement par le bout de line supplémentaire et plus du tout par le gap. Ceci devrait encore améliorer la linéarité.


(a)

Fig. 6 Mesure (a) de la phase de $S_{21}$ et (b) de l'amplitude de $S_{21}$ et de $S_{11}$ sous différentes conditions d'éclairement.

Notons que tout type de coupleur convient et peut être transformé en quadripôle déphaseur. Il suffit d'ajouter les deux tronçons de ligne après un gap sur deux bras correctement choisis et d'éclairer ces gaps de façon adéquate pour obtenir le fonctionnement désiré. Un coupleur $-3 \mathrm{~dB}$ à lignes parallèles [12] a montré un fonctionnement semblable à celui présenté pour le coupleur en anneau de Wilkinson.

\section{CONCLUSION}

Le contrôle optique trouve un intérêt évident dans la réalisation de dispositifs hyperfréquences reconfigurables dans la mesure où la fréquence d'utilisation des dispositifs est suffisamment élevée pour que les pertes induites par le plasma photoinduit soient comparables à celles de moyens de commande plus classiques tels que les diodes varicap, c'est-à-dire au-delà de $30 \mathrm{GHz}$. Pour les études menées dans le cadre de ce papier, les gammes de fréquence sont plus basses. En faisant donc abstraction des pertes, nous avons montré la faisabilité d'un déphaseur hyperfréquence permettant de conserver un coefficient de transmission constant tout en faisant varier de façon continue la phase entre 0 et $90^{\circ}$ à $10 \mathrm{GHz}$. Il est à noter de plus que ce déphaseur est large bande dans la mesure où la variation de phase reste quasiment identique sur une bande de $500 \mathrm{MHz}$ autour de la fréquence centrale.

\section{REMERCIEMENTS}

Les auteurs remercient Olivier Exshaw pour son aide précieuse quant à la réalisation des mesures hyperfréquences.

\section{RÉFÉRENCES}

[1] Sangi Lee, Yasuo Kuga, and R. A. Mullen, "Optically tunable, millimeter-wave attenuator based on layered structures", Microwave Opt. Technol. Lett., vol. 27, $\mathrm{N}^{\circ}$ 1, Oct 2000, pp 9-13.

[2] W. Platte, "Periodic-structure Photoexcitation of a Silicon Coplanar Waveguide for Selective Optoelectronic Microwave control", IEEE Trans Microwave Theory Tech ., vol. 38, $\mathrm{n}^{\circ}$ 5, pp. 638646, May 1990.

[3] A. Safwat, D. Khalil, H. Elhennawy, and H. Ragaie, "Quasi-static Analysis of an Optically Illuminated Directional Coupler", IEEE Trans Microwave Theory Tech, vol. 45, Aug. 1997, pp. 1351-1357.

[4] P. Cheung, D. Neikirk, and T. Itoh, "Optically Controlled Coplanar Waveguide Phase-Shifter", IEEE Trans Microwave Theory Tech ., vol 38, pp. 586-594, May 1990.

[5] C.H. Lee, P.S. Mak, and A.P. DeFonzo, "Optical Control of Millimetrewave Propagation in Dielectric Waveguide", IEEE J Quantum Electron, vol. 16, 1980, pp. 277.

[6] M. El Khaldi, F. Podevin, J-D Arnould, A. Vilcot, "Conception et modélisation d'un déphaseur microruban à base d'un coupleur résonateur directionnel à deux gaps commandé par un signal optique continu", $3^{\text {èmes }}$ Journées FrancoMaghrébines des Micro-ondes et leurs Applications, Marrakech, 15-17 octobre 2003.

[7] SILVACO, Atlas user's manual, device simulation software, edition 2002.

[8] R.Gary, J.-D. Arnould, and A. Vilcot, "3Dimensions Analytical Modelling of the Optical Carriers Injection in a Semiconductor Substrate", MAPE'2005 conference, Beijing, China

[9] MATLAB ${ }^{\mathrm{TM}}$, User's manual, The MathWorks, Inc., 2004.

[10] CST Microwave Studio, User's guide, edition 2003.

[11]J. Reed and G. J. Wheeler, "A Method Of Analysis of Symmetric Four Ports Networks", IRE Trans. On Microwave Theory and Tech., October 1956, pp. 246-252.

[12] M. El Khaldi, F. Podevin and A. Vilcot, "Microstrip parallel-line coupler to perform broadband optically controlled phase-shifting", Microwave Opt. Technol. Lett. Vol. 47, $\mathrm{N}^{\circ}$ 6, Dec. 2005, pp 570-573. 